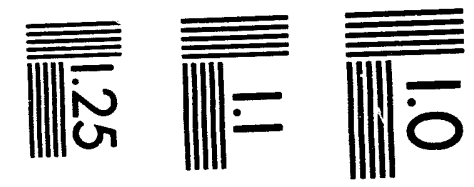

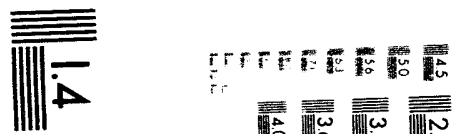

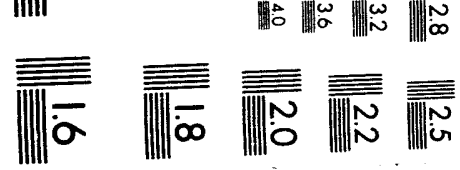



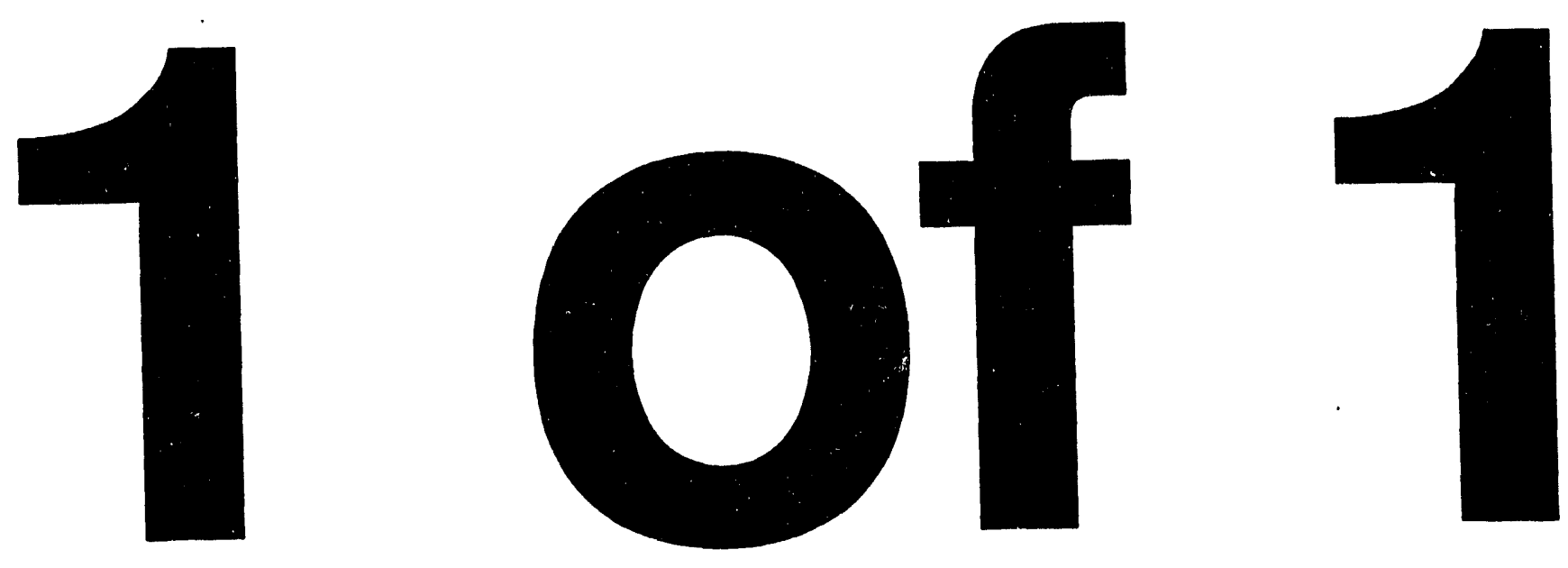


\section{Conf $-931234--5$}

LA-UR- 93-4477

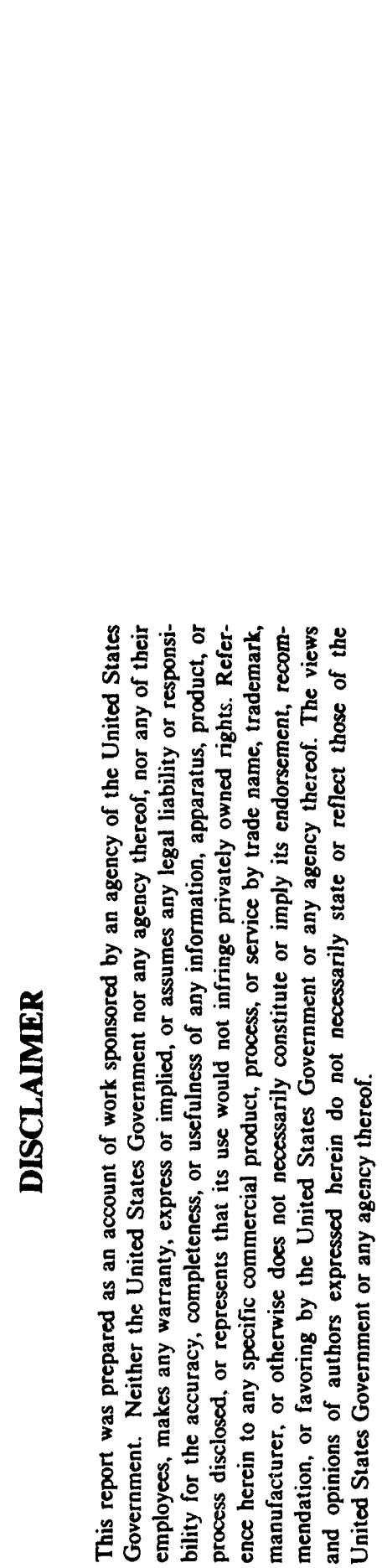

RESONANT ULTRASOUND SPECTROSCOPY FOR ELASTIC

CONSTANT MEASUREMENTS

Author(s):

R. D. Dixon, MTL-6

A. Migliori, MTL-10

L. H. Roe, MTL-6

Submitted to:

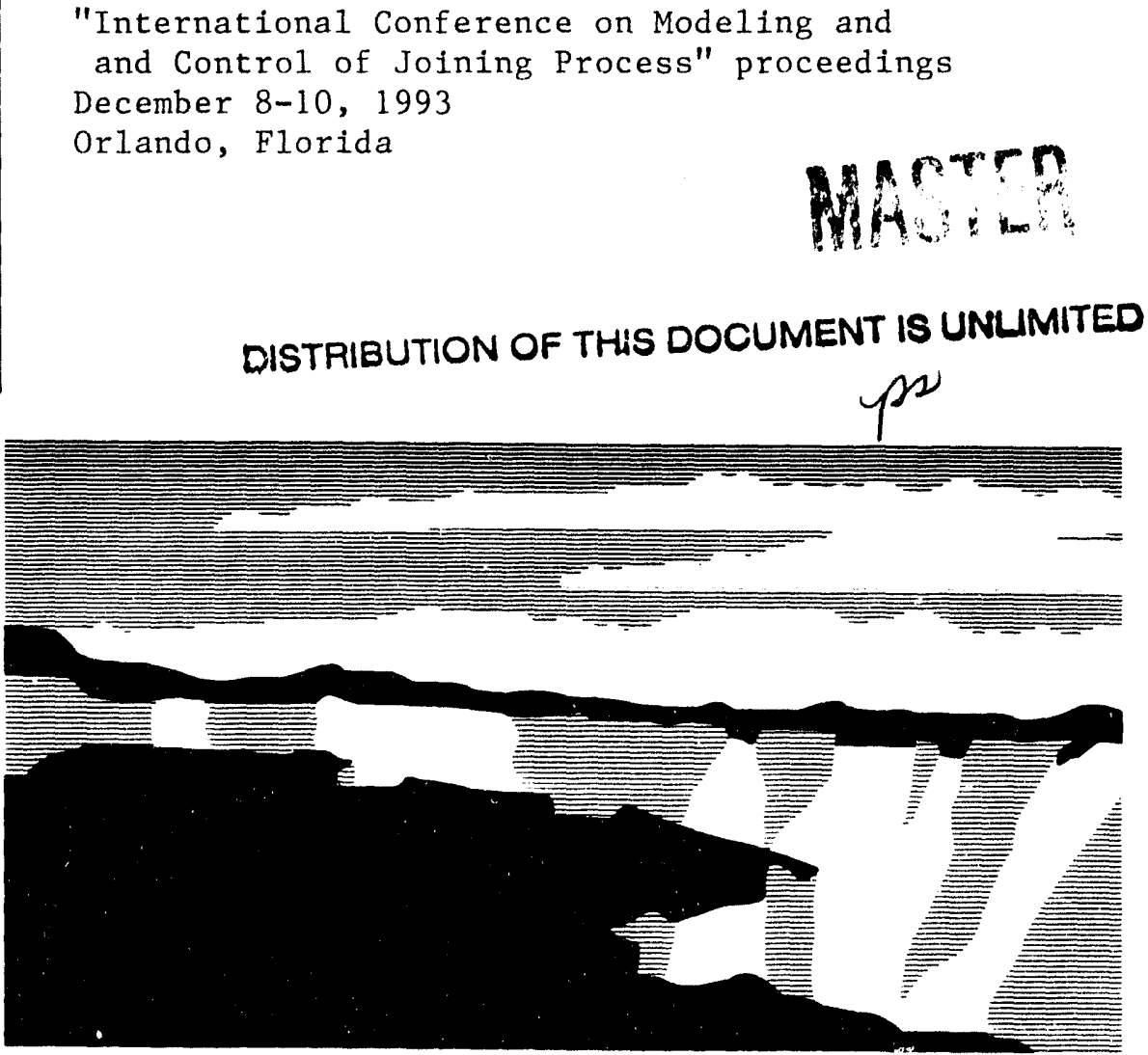

Los Alamos National Laboratory, an affirmative action/equal opportunity employer, is operated by the University of California for the U.S. Department of Energy under contract W-7405-ENG-36. By acceptance of this article, the publisher recognizes that the U.S. Government retains a nonexclusive, royalty-free license to publish or reproduce the published form of this contribution, or to allow others to do so, for U.S. Government purposes. The Los Alamos National Laboratory requests that the publisher identify this articte as work performed under the auspices of the U.S. Department of Energy. 


\title{
RESONANT ULTRASOUND SPECTROSCOPY FOR ELASTIC CONSTANT MEASUREMENTS
}

\author{
R. D. Dixon*, A. Migliori*, Larry Roe*
}

\begin{abstract}
All objects exhibit vibrational resonances when mechanically excited. These resonant frequencies are determined by density, geometry, and elastic moduli. Resonant ultrasound spectroscopy (RUS) takes advantage of the known relationships between the parameters. In particular, for a freely suspended object, with three of the four parameters (vibrational spectra, density, geometry, or elastic moduli) known the remaining one can be calculated. From a materials characterization standpoint it is straight-forward to measure density and geometry but less so to measure all the elastic mouuli. It has recently become possible to quickly and accurately measure vibrational spectra, and using code written at Los Alamos, calculate all the elastic mo uli simultaneously. This is done to an accuracy of better than one perrent for compression and 0.1 percent for shear.
\end{abstract}

RUS is based on the Hamiltonian approach for calculating resonance modes of elastic bodies having free boundaries in which minimization of the Lagrangian converts a volume calculation to a surface one. This greatly reduces the computational time while improving accuracy. The approach is only viable for simple geometric shapes. The objects vibrational resonances are both excited and detected using modified piezoelectric transducers. The excitation is done using a swept sine wave signal generator with a heterodyne receiver both developed at Los Alamos National Laboratory. To date RUS has the highest absolute accuracy of any laboratory diagnostic modulus measurement system, and it is applicable to samples with a largest dimension of $1 \mathrm{~mm}$.

RUS provides rapid acquisition of materials information here-to-fore obtainable only with difficulty. It will greatly facilitate the use of real materials properties in models and thus make possible more realistic modeling results. The technique is sensitive to phase changes and microstructure. This offers a chance to input real data into microstructure and phase change models. It will also enable measurement of moduli at locations in and about a weld thus providing information for or validating coupled thermomechanical calculations.

\section{INTRODUCTION}

Resonant ultrasound spectroscopy (RUS) is the measurement and analysis of vibrational resonances to determine mechanical properties of an object. For example, elastic moduli or the existence of a defect can be determined. The mathematical basis for the analysis (Ref. 1,2) is a simple variational principle which is used to derive an eigenvalue equation for the normal-mode frequencies and eigenvectors for the vibrations of an elastic body with free boundaries. The work was extended (Ref. 3-5) and improved on (Ref. 6, 7) such that it is now possible to accurately measure and analyze resonance frequencies to determine elastic moduli (Ref. 8) by non-expert users. The mathematical background, electronics requirements, transducers, data, and

* Materials Division, Los Alamos National Laboratory, PO Box 1663, Los Alamos, NM 87544. 
results will be presented here. Some of this has been presented elsewhere but is presented here for completeness and the results are extended.

\section{ANALYSIS}

Following (Ref. 7), a Lagrangian (L) approach is used to solve the resonance problem for an arbitrarily shaped body with volume $\mathrm{V}$, elastic tensor $\mathrm{C}_{\mathrm{ijk} \text { l }}$, and density $\rho$,

$$
L=\int(K E-P E) d V \text {. }
$$

The kinetic energy (KE) is given by;

$$
\mathrm{KE}=\frac{1}{2} \rho \omega^{2} \mathrm{U}_{\mathrm{i}}^{2}
$$

The potential energy (PE) is given by;

$$
\mathrm{PE}=\frac{1}{2} \mathrm{C}_{\mathrm{ijk}, \mathrm{U}} \mathrm{U}_{\mathrm{i}, \mathrm{j}} \mathrm{U}_{\mathrm{k}, 1}
$$

with

$$
\mathrm{U}_{\mathrm{i}, \mathrm{j}}=\frac{\partial \mathrm{U}_{\mathrm{i}}}{\partial \mathrm{X}_{\mathrm{j}}}
$$

In the above equations $U_{i}$ is the ith component of the displacement vector, $\omega$ the frequency, the usual summation convention applies, and indices following a comma denote differentiation with respect to that coordinate.

Using the elastic wave equation,

$$
\rho \omega^{2} U_{i}+C_{i j k l} U_{k .1 j}=0
$$

and the vanishing of the ith component of the surface traction vector,

$$
\mathrm{n}_{\mathrm{i}} \mathrm{C}_{\mathrm{ijk} \mathrm{l}} \mathrm{U}_{\mathrm{k}, \mathrm{l}}=0
$$

where $n_{i}$ is the unit outer normal to the surface $S$, we have with the variational principle

$$
\delta \mathrm{L}=\int_{V} \operatorname{lhs}(5)_{i} \delta_{i} d V+\int_{s} \operatorname{lhs}(6)_{i} \delta_{i} d S
$$

In equation $7, \mathrm{lhs}()$ refers to left hand side of the corresponding equations. When $\delta L=0, \omega$ is a normal mode vibrational frequency of the system eigenvalue, and is determined by both pieces of equation 7 being independently zero. Thus, using the surface integral instead of the volume integral produces the desired answer. 
Following Rayleigh-Ritz, expand the displacement vector in a complete set of functions $\left\{\Phi_{\lambda}\right\}$,

$$
\mathrm{U}_{\mathrm{i}}=\mathrm{a}_{\lambda i} \Phi_{\lambda}
$$

and choose powers of the Cartesian coordinates as the basis functions:

$$
\Phi_{\lambda}=x^{\prime} y^{m} z^{n}
$$

$\lambda=(1, \mathrm{~m}, \mathrm{n})$ is the function label which is a set of three nonnegative integers. After appropriate substitutions:

$$
L=\frac{1}{2} \omega^{2} a^{T} E a-\frac{1}{2} a^{T} \Gamma a
$$

where $\mathrm{E}$ and $\Gamma$ are matrices whose order $\mathrm{R}$ is determined by the truncation condition

$$
1+m+n \leq N
$$

with $R=3(N+1)(N+2)(N+3) / 6$. Migliori et. al. found that $N=10$ gives a good compromise between computational accuracy, computing time and sample preparation errors consistent with data spanning the first 50 modes. For the above transformations note that $\{a\}$ becomes a column vector and $\mathrm{a}^{\mathrm{T}}$ its transpose. The matrix elements for $\mathrm{E}$ and $\Gamma$ are:

$$
E_{\lambda i \lambda^{\prime i}}=\delta_{i i^{\prime}} \int_{V} \Phi_{\lambda} \rho \Phi_{\lambda}, \mathrm{dV} \text { and } \Gamma_{\lambda i \lambda^{\prime} i^{\prime}}=\mathrm{C}_{\mathrm{iji} i^{\prime}{ }^{\prime}} \int_{\mathrm{V}} \Phi_{\lambda, j} \Phi_{\lambda \cdot, j} \mathrm{dV} \text {. }
$$

For solutions, $\mathrm{U}_{\mathrm{i}}$, to the free-vibration problem, solutions to equation 10 are found by differentiating with respect to each of the $\mathrm{R}$ amplitudes and equating to zero. This yields the following:

$$
\omega^{2} \mathrm{Ea}=\Gamma \mathrm{a} .
$$

$E$ and $\{a\}$ are symmetric and positive definite and $\Gamma$ is symmetric so that a standard eigenvalueeigenvector subroutine package (RSG in EISPACK-Ref. 9) can be used to solve 13.

The inverse problem, namely determination of the elastic moduli given the eigenfrequencies, is solved by differentiating equation 13 with respect to sample parameters ( $p$ ) and having a defined figure of merit. The figure of merit is the sum of squares of the differences between the measured and calculated parameters. This yields the following equation:

$$
\frac{\partial \omega^{2}}{\partial p} E a+\omega^{2} E \frac{\partial a}{\partial p}+\omega^{2} \frac{\partial E}{\partial p} a=\frac{\partial \Gamma}{\partial p} a+\Gamma \frac{\partial a}{\partial p} .
$$

Multiplying on the left by $\mathrm{a}^{\mathrm{T}}$ and comparing with the transpose of equation 13 gives: 


$$
\frac{\partial \omega^{2}}{\partial p}=\left(a^{T}\left[\frac{\partial \Gamma}{\partial p}-\omega^{2} \frac{\partial E}{\partial p}\right] a\right) .
$$

The eigenvectors $\{\mathrm{a}\}$ have been calculated and the volume integrals for $\partial \Gamma / \partial \mathrm{p}$ and $\partial \mathrm{E} / \partial \mathrm{p}$ are straight forward and do not represent a major increase in computation. One can take advantage of symmetry to reduce the computation time for both the eigenvalue equation and the inverse problem. These details are presented in Ref. 7.

The inverse problem, while not readily tractable is best handled by weighted least squares using a Levenberg-Marquardt (Ref. 10) minimization scheme. As discussed in Ref. 7, this scheme allows determination of the compressional moduli $\left(c_{\mathrm{ii}}, \mathrm{i}=1,3\right)$ to less than $1 \%$, the shear moduli $\left(c_{i i}, i=4,6\right)$ to less than $0.02 \%$, and off-diagonal moduli to less than $3 \%$. This is readily accomplished using the electronics and transducers described below provided the sample geometry is accurate to a few $\mu \mathrm{m}$ or better for a $1 \mathrm{~mm}$ rectangular parallelepiped.

For a purely isotropic, and homogenous material we can further write the engineering parameters Young's modulus ( $Y$ ), Poisson's ratio $(v)$, bulk modulus $(K)$, shear modulus $(G)$, and the Lame constants $(\lambda$ and $\mu)$ in terms of the elastic moduli as:

$$
\begin{aligned}
& \lambda=C_{12} \\
& v=\frac{C_{12}}{\left(C_{12}+C_{11}\right)} \\
& \mu=G=C_{44} \\
& 2 C_{44}=C_{11}-C_{i 2} \\
& Y=2 C_{44}\left(C_{11}+2 C_{12}\right) /\left(C_{11}+C_{12}\right) \\
& K=\left(2 C_{44} / 3\right)\left(C_{11}-2 C_{12}\right) /\left(C_{11}-C_{12}\right)
\end{aligned}
$$

For objects exhibiting less symmetry these relations can not be simply stated and one considers the clastic moduli only.

\section{ELECTRONICS}

The small transducer signals and low contact force require the best possible signal-to-noise ratio for the data acquisition electronics, to assure detection of as many vibrational modes as possible. 
Because of the high mechanical $Q$ (typically $10^{5}$ ) and the improvement in efficiency, we designed and use a swept sine approach based on a heterodyne receiver for the excitation and detection of resonances. A complete description of the circuits and elements can be found in Ref. 7. The electronics is available commercially from Quatro Corp. (Ref. 11). A Quatro electronics system was used for the work reported here.

\section{TRANSDUCERS}

RUS requires excitation and detection of the natural vibrational resonances of an object. The frequencies of interest are in the acoustical region which allows the use of ultrasonic transducers. The transducers required must be sensitive but more importantly, must not add or fail to detect lines in the spectra. The latter possibililics result if resonances exist at frequencies where the transducers have no sensitivity or they ring. For most commercial ultrasonic transducers the placement of the piezoelectric element, methods of mounting the various attachments, and the attachments produce many resonances. These create extra lines in the spectra and eliminate many others. The result is a spectra with more uncertainty than can be accepted for accurate RUS measurement of material properties.

We have overcome this by fabricating our own transducers (patent applied for) which significantly reduces the above effects. The result is a transducer which has only weak, low $Q$ resonances in the range of 0 to $10 \mathrm{MHz}$. This has been verified by measuring the transducers electrical response with a network analyzer and also by a capacitance bridge method. We plan future activities to further characterize the transducer. Figure 1 shows the capacitance bridge output from a bare piezoelectric element (PZT-5A) and one of our transducers. This figure shows the lack of resonances in the range 0 to $3 \mathrm{MHz}$. Because these transducers do not have resonances in the region of interest they provide spectra representative of the object under test and one is confident that the lines should fit the model and yield real elastic moduli.

\section{RESULTS}

Initial work was done using aluminum alloy 7075 in the as received and welded conditions. Table I shows the handbook chemistry and Table II shows other properties of this alloy. For this work two specimens were used. One from the base metal (BM) and one completely from an autogeneous electron beam weld (WM). Depending upon its structure, individual vibrational resonances of a cube may be degenerate. Thus, for simplicity and uniqueness, all samples are prepared as rectangular parallelipipeds with the dimensions differing by a few percent. Also, slight deviations in parallelism or perpendicularity of the faces cause modes to shift and thus prevent fitting the measured resonances to the calculated data. For these reasons sample preparation is critical. We machine ground the samples to dimensions of about $0.3 \mathrm{~cm} \pm 0.0002$ $\mathrm{cm}$. This gave good results as the data shows. Additionally we found that because the samples were smaller than the transducers (diagonal cross section versus transducer diameter) there were gas resonances in the spectrum. These were eliminated by making the measurements in a vacuum. Figure 2 is a typical RU spectrum. 


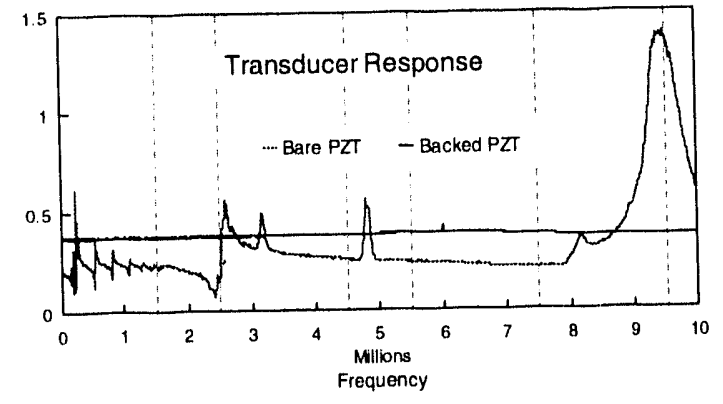

Figure 1. Difference in response between a bare PZT piezoelectric crystal and the new transducers.

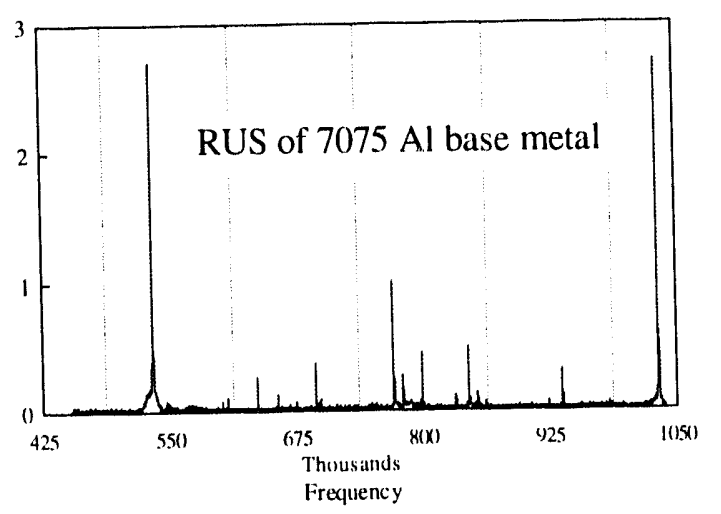

Figure 2. Resonant ultrasound spectrum from 7075 aluminum base metal.

TABLE I. 7075 aluminum composition.

\begin{tabular}{|l|l|l|l|l|l|l|l|l|}
\hline $\mathrm{Cu}$ & $\mathrm{Mg}$ & $\mathrm{Mn}$ & $\mathrm{Si}$ & $\mathrm{Fe}$ & $\mathrm{Cr}$ & $\mathrm{Zn}$ & $\mathrm{Ti}$ & Other \\
\hline $1.2-2.0$ & $2.1-2.9$ & $0.3 \max$ & $0.4 \max$ & $0.5 \max$ & $.18-0.28$ & $5.1-6.1$ & $0.2 \mathrm{max}$ & .05 each \\
\hline
\end{tabular}

TABLE II. 7075 aluminum mechanical properties.

\begin{tabular}{|l|l|}
\hline Poisson's ratio & 0.33 \\
\hline Elastic modulus in tension & $10.3 \times 10^{6} \mathrm{PSI}$ \\
\hline Elastic modulus in compression & $10.5 \times 10^{6} \mathrm{PSI}$ \\
\hline Shear modulus & $3.9 \times 10^{6} \mathrm{PSI}$ \\
\hline
\end{tabular}

Spectra similar to that shown in Figure 2 were analyzed to determine the elastic constants. Initially the spectra were analyzed assuming the specimen was isotropic and homogeneous (two independent elastic moduli). This resulted in a reasonable fit but several calculated lines differed excessively from the measured lines. The data were then fit assuming an orthorhombic structure ( 9 independent elastic moduli). For the BM this produced an excellent fit. (For all of this work, goodness of fit was determined by the rms error of the least square fit to the data. An rms error of less than $0.2 \%$ is considered excellent and produces moduli with less than $1.0 \%$ error. Additional considerations are that each calculated line be within $0.5 \%$ of the measured line and that no lines are missing.) The orthorhombic fit for the BM met all of these considerations. This implies that the material is textured and a fit assuming hexagonal elastic moduli ( 5 independent elastic moduli) would be better. For the base metal this was not the case. However, the present analysis routine assumes the principal axes are oriented along the sample edge. This implies that since an orthorhombic fit of the elastic moduli is best, the material may be textured along an axis not coincident with the sample edge. Work is underway to modify the analysis routine to accommodate principal axes oblique to the sample edge. Table III shows the output from the orthorhombic fit for the BM. The analysis also allows a best fit to be obtained by adjusting the dimensions or holding them constant at the measured values. Both cases were calculated for these samples. In all cases, as one might expect, the best fit was obtained by allowing the dimensions to be fit. The difference between the measured and fit dimensions are shown in Tables IV and V. Also note in Table III columns for wt, $\mathrm{k}, \mathrm{i}$, and $\mathrm{df} / \mathrm{dc}_{\mathrm{ij}}$. Wt is the weight given 
to each measured line, one (1) means that the value of the measured line is used while zero (0) means that it is not used but that a line exists in the spectra in that position. The $\mathrm{k}$ and $\mathrm{i}$ refer to the mode identification as outlined in (Ref. 7). The $\mathrm{df} / \mathrm{dc}_{\mathrm{ij}}$ represent the change in frequency for the given $\mathrm{C}_{\mathrm{ij}}$. These last data can be used to assure that the modes are adequately represented (i.e. the spectra is not composed entirely of shear or compressional modes, which would be unrealistic). Table IV shows the elastic moduli, fit dimensions, and the percent changes for each moduli which produce a $2.0 \%$ change in the chisquare error for the analysis. The error shown for the dimensions is the percent difference between the measured and fit values.

A similar analysis was done for the WM. This analysis resulted in a best fit for the hexagonal case. Since one of the sample axes was the welding direction one assumes that the sample is textured and that the texture axis is parallel or perpendicular to the welding direction. This will be verified by fitting the principal axes to sample orientation. The elastic moduli, dimensions, and errors for the WM are shown in Table V. Comparing individual moduli suggest that the weld metal softened by welding. However, comparisons will not be done until the full analysis can be done.

We have shown that this technique is sensitive to the effects of welding and will provide data that can be used for thermomechanical modeling. Further improvements will allow some limited temperature data to be obtained which will further enhance the modeling and its interpretation.

\section{REFERENCES}

1. Holland, R. $1968 . \quad$ J. Acoust. Soc. Am. 43: 988-.

2. Demarest, H. H. 1971 . J. Acoust. Soc. Am. 49: 768-.

3. Ohno, I. $1976 . \quad$ J. Phys. Earth 24: 355-.

4. Ohno, I. 1990. Chem. Minerals 17: 371-.

5. Anderson, O. L., Schreiber, E. , and Soga, N. 1973. Elastic moduli and Their Measurements: New York: McGraw-Hill.

6. Visscher, W. M.; Migliori, A.; Bell, T. M.; and Reinert, R. A. 1991. On the Normal Modes of Free Vibration of Inhomogeneous and Anisotropic Elastic Objects. J. Acoust. Soc. Am. 90 (4): 2154-2162.

7. Migliori, a.; Sarrao, J. L.; Visscher, W. M.; Bell, T. M.; Lei, M.; Fisk, Z.; and Leisure, R. G. 1993. Resonant Ultrasound Spectroscopic Techniques for Measurement of the Elastic Moduli of Solids. Physica B. 183: 1-24.

8. Lei, M.; Sarrao, J. L.; Visscher, W. M.; Bell, T. M.; Thompson, J. D.; and Migliori, A. 1993. Elastic Constant of a Monocrystal of Superconducting $\mathrm{YBa}_{2} \mathrm{Cu}_{3} \mathrm{O}_{7-\delta}$. Physical Rev. B 47 (10): 6154-6156.

9. Smith, B. T.; Boyle, J. M.; Dongarra, J. J.; Garbow, B. S.; Ikebe, Y; Klema, V. C. ; and Moler, C. B. 1976. Matrix Eigensystem Routines - EISPACK Guide, Lecture Notes in Computer Science No. 6. eds. Goos, B and Hartmanis, J. New York, Springer. Garbow, B. S.; Boyle, J. M.; Dongarra, J. J.; and Moler, C. B.; 1977. Matrix Eigensystem Routines EISPACK Guide Extension, Lecture Notes in Computer Science No. 51. eds. Goos, G. and Hartmanis, J. New York, Springer.

10. Press, W. H; Lannery, B. P.; Teukolsky, S. A.; adn Vetterling, W. T. 1986. Numerical Recipes. Cambridge University Press.

11. Quatro Corporation, 4300 San Mateo NE, Suite B-2890, Albuquerque, NM 87110. 
TABLE III. Table of output from RUS analysis using orthorhombic symmetry for 7075 aluminum base metal.

\begin{tabular}{|c|c|c|c|c|c|c|c|c|c|c|c|c|c|c|c|}
\hline & Freq $_{\text {mea }}$ & Freq $_{c a l}$ & $\%$ diff & $\mathrm{wt}$ & $\mathrm{k}$ & 1 & $\mathrm{df} / \mathrm{dc}_{11}$ & $\mathrm{df} / \mathrm{dc}_{32}$ & $d f / d c_{33}$ & $\mathrm{df} / \mathrm{dc}_{93}$ & $\mathrm{df} / \mathrm{dc}_{13}$ & $\mathrm{df} / \mathrm{dc}_{12}$ & $\mathrm{df} / \mathrm{dc}_{41}$ & $\mathrm{df} / \mathrm{dc}_{55}$ & $\mathrm{df} / \mathrm{dc}_{6 \mathrm{ft}}$ \\
\hline 1 & 717 & 0.370133 & -0.42 & 1 & 4 & 1 & 0.01 & 0.01 & 0.02 & -0.01 & -0.01 & 0 & 0.31 & 0.05 & 0.62 \\
\hline 2 & 467 & & & 1 & 4 & 2 & 0.02 & & 0.01 & & 0 & -0.0 & 0.34 & 0.59 & 0.04 \\
\hline 3 & & & $z$ & 1 & 6 & 1 & 0.6 & 1.02 & 0.02 & -0.14 & 0.09 & -0.8 & 0.15 & .05 & 0 \\
\hline 4 & & & 0 & 1 & 1 & 1 & 0.06 & 1.13 & & 69 & A & -0.25 & 0 & .02 & 0.2 \\
\hline 5 & & & 0 & 1 & 3 & 2 & 0.03 & 0.05 & 0.03 & -0.03 & -0.02 & 0 & 0 & 0 & 0.94 \\
\hline 6 & & & & 1 & 8 & 1 & 0.06 & 0.07 & 0.02 & 0 & -0.02 & -0.06 & 0.92 & 0 & 0 \\
\hline 7 & & & -0 & 1 & 7 & 1 & 1.06 & 0.03 & 0.57 & 0.11 & -0.8 & -0.16 & $0.0^{2}$ & 0 & 0.15 \\
\hline 8 & & & -0 . & 1 & 2 & 2 & 0.07 & 0.1 & 0.04 & -0.05 & 0.01 & -0.07 & 0 & 0.9 & 0 \\
\hline 9 & & & & 1 & 5 & 1 & 0.74 & 1.31 & 0.06 & -0.28 & 0.2 & -1.03 & 0 & 0 & 0 \\
\hline 10 & & & $-C$ & 1 & 6 & 2 & 0.59 & 0.16 & 0.27 & -0.15 & -0.37 & 0.1 & 0.15 & 0.24 & 0.01 \\
\hline 11 & & & & 1 & 5 & 2 & & 0.74 & 73 & -0.46 & -0.36 & -0.27 & 0 & & 0 \\
\hline 12 & & & & 1 & 2 & 3 & 0.11 & 0.87 & 0.09 & -0.29 & 0.1 & -0.32 & 0.03 & 0.39 & 0.02 \\
\hline 13 & & & & 1 & 5 & 3 & 0. & 0.1 & 17 & -0.3 & -1 & 0.22 & 0 & 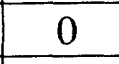 & 0 \\
\hline 14 & 79 & & -0. & 1 & 8 & 2 & & 4 & 1 & 0.12 & -0.32 & -0.39 & 0.29 & 0.06 & 0.02 \\
\hline 15 & & & & 1 & 1 & 2 & 0 & 0.07 & 0.76 & -0.02 & -0.41 & -0.04 & 0.01 & 0.25 & 0.12 \\
\hline 16 & & & -0.0 & 1 & 7 & 2 & 0 & 3 & 0.6 & -0.39 & 9 & 14 & 4 & 01 & 0.15 \\
\hline 17 & & & & 1 & 3 & 3 & & 0.16 & 1.12 & -0.44 & -0.38 & 0.14 & 0.01 & 0.04 & 0.23 \\
\hline 18 & & & -0 & 1 & 2 & 4 & 0. & 0.11 & 0.09 & -0.06 & -0.05 & -0.03 & 0.42 & 03 & .41 \\
\hline 19 & 72 & & & 1 & 5 & 4 & 0. & 0.82 & 0.34 & -0.24 & 0.09 & -0.62 & 0.03 & 0.02 & 0.01 \\
\hline 20 & & & & 1 & 4 & 3 & 0.03 & 0.04 & 0.02 & -0.01 & -0.01 & -0.01 & 0.31 & 0.35 & 0.28 \\
\hline 21 & 4 & 44 & 0 & 1 & 8 & 3 & 0.18 & 0.13 & 3 & -0.07 & -0.09 & -0.06 & 0.04 & 0.37 & 0.38 \\
\hline 22 & & & & 1 & 6 & 3 & 0.67 & 0.56 & & -0.14 & -0.21 & -0.38 & 0.16 & 0.11 & 0 \\
\hline 23 & & & & 1 & 7 & 3 & 0 & 0.45 & 0.62 & -0.3 & -0.28 & -0.28 & 0.07 & 0 & 0.12 \\
\hline 24 & 0.8133 & & -0 & 1 & 5 & 5 & 6 & 0.19 & 1.01 & -0.31 & -0.64 & 0.14 & 0 & 0 & 0.03 \\
\hline 25 & & & & 1 & 1 & 3 & 0.35 & 0.54 & 0.66 & -0.3 & -0.29 & -0.2 & 0 & 0.07 & 0.16 \\
\hline 26 & & & & 1 & 3 & 4 & 0.2 & 0.2 & 0.23 & -0.1 & -0.12 & -0.12 & 0.34 & 0.32 & 0.04 \\
\hline 27 & 0.8736 & 0.872957 & -0.07 & 1 & 1 & 4 & 0.2 & 0.48 & 0.19 & -0.21 & 0.07 & -0.27 & 0 & 0.09 & 0.46 \\
\hline
\end{tabular}




\begin{tabular}{|c|c|c|c|c|c|c|c|c|c|c|c|c|c|c|c|}
\hline & & & di & & & & $\mathrm{ac}$ & $70 \mathrm{c}$ & $1 / \mathrm{d}$ & $/ \mathrm{d}$ & $\mathrm{u}_{1}$ & 0,12 & $100_{44}$ & - & 10066 \\
\hline 28 & & 22 & 0.09 & 1 & & 4 & 0.55 & 0.2 & 0.28 & 0.09 & -0.32 & -0.26 & 0.11 & 0 & 0.35 \\
\hline 29 & & & .07 & 1 & 0 & 4 & 0.13 & 0.39 & & 8 & 5 & 1 & - & 0.1 & 0 \\
\hline 30 & & & & 1 & 7 & t & 0.1 & - & & -0 & 0.14 & -0.19 & .23 & 0 & .03 \\
\hline 31 & & & .02 & 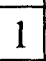 & 1 & 5 & 0.6 & 05 & 0.6 & 0. & -0.66 & -0.12 & 0 & 0.3 & 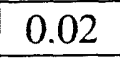 \\
\hline 32 & & & & 1 & 6 & 5 & 0.43 & 0.13 & 0.57 & -0.23 & -0.5 & 0.17 & 0.08 & 0. & .01 \\
\hline 33 & & & 0.08 & 1 & 6 & 6 & 0.16 & 0.18 & 0. & -0.05 & -0.0 & 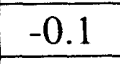 & 0.21 & 3 & .26 \\
\hline 34 & & & 0.03 & 1 & 3 & 5 & 0.28 & 38 & & & 05 & & 0.04 & 0.0 & 0.48 \\
\hline 35 & & & & -4 & & $\pi$ & 0. & 0.43 & 0.17 & -0.14 & -0.04 & -0.19 & 0.44 & 0.1 & .05 \\
\hline 36 & & & 07 & 1 & 4 & 4 & 0.29 & 0.32 & 0 & -0.12 & -0.13 & -0 & .26 & 1 & 24 \\
\hline 37 & & & & 1 & & 2 & 0. & 0.28 & & -0.09 & 0.03 & -0.24 & 0.31 & 0.16 & 0.2 \\
\hline 38 & & & & -1 & & 6 & 0 & 0.19 & 0 & 01 & -0.07 & -0.07 & .25 & 0.3 & 0.19 \\
\hline 39 & & & & 1 & & 4 & 0.3 & 13 & & & 31 & & 0.15 & 0.02 & 0.31 \\
\hline 40 & & & & 1 & 3 & 6 & 0 & 0.23 & & -0.28 & -0.23 & 7 & 0.19 & .22 & 0.12 \\
\hline 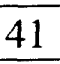 & & & & 1 & & 5 & 0.3 & .2 & 02 & 001 & -0.17 & -0.16 & .22 & 25 & 0.16 \\
\hline 42 & & & & 1 & & 7 & 0.36 & 0.37 & 0.43 & -0.12 & -0.02 & -0.2 & .05 & .03 & 0.1 \\
\hline 43 & & & & 1 & 2 & 6 & & 0.13 & & & -0.19 & -0.05 & 4 & .45 & 0.1 \\
\hline 44 & 1.08912 & 1.090487 & 0.13 & 1 & 7 & 6 & 17 & 0.15 & 0.18 & -0.08 & -0.09 & -0.08 & 0.26 & 0.25 & 0.22 \\
\hline
\end{tabular}

TABLE IV. Table of output from RUS analysis for 7075 aluminum base metal. Analysis is for orthorhombic symmetry.

\begin{tabular}{|c|c|c|c|c|c|c|c|c|c|c|c|c|}
\hline Parameter & $\mathrm{C}_{11}$ & $\mathrm{C}_{22}$ & $\mathrm{C}_{33}$ & $\mathrm{C}_{23}$ & $\mathrm{C}_{13}$ & $\mathrm{C}_{12}$ & $\mathrm{C}_{44}$ & $\mathrm{C}_{55}$ & $\mathrm{C}_{66}$ & $\mathrm{~d}_{1}$ & $\mathrm{~d}_{2}$ & $\mathrm{~d}_{3}$ \\
\hline value & 1.115 & 1.1246 & 1.1178 & 0.5867 & 0.5822 & 0.5884 & 0.2672 & 0.2677 & 0.2708 & 0.34945 & .37793 & 0.3188 \\
\hline error & 0.53 & 0.68 & 0.43 & 1.02 & 0.75 & 1.06 & 0.11 & 0.09 & 0.1 & 0.07 & 0.01 & 0.06 \\
\hline
\end{tabular}

TABLE V. Table of output from RUS analysis for 7075 aluminum weld metal. Analysis is for hexagonal symmetry.

\begin{tabular}{|c|c|c|c|c|c|c|c|c|c|c|c|c|}
\hline Parameter & $\mathrm{C}_{11}$ & $\mathrm{C}_{22}$ & $\mathrm{C}_{33}$ & $\mathrm{C}_{23}$ & $\mathrm{C}_{13}$ & $\mathrm{C}_{12}$ & $\mathrm{C}_{44}$ & $\mathrm{C}_{55}$ & $\mathrm{C}_{66}$ & $\mathrm{~d}_{1}$ & $\mathrm{~d}_{2}$ & $\mathrm{~d}_{3}$ \\
\hline value & & & 1.0798 & 0.5472 & & 0.5517 & 0.2687 & & 0.265 & 0.37534 & .34754 & 0.32047 \\
\hline error & & & 0.51 & 1.06 & & 1.05 & 0.06 & & 0.17 & 0.02 & 0.02 & 0.1 \\
\hline
\end{tabular}



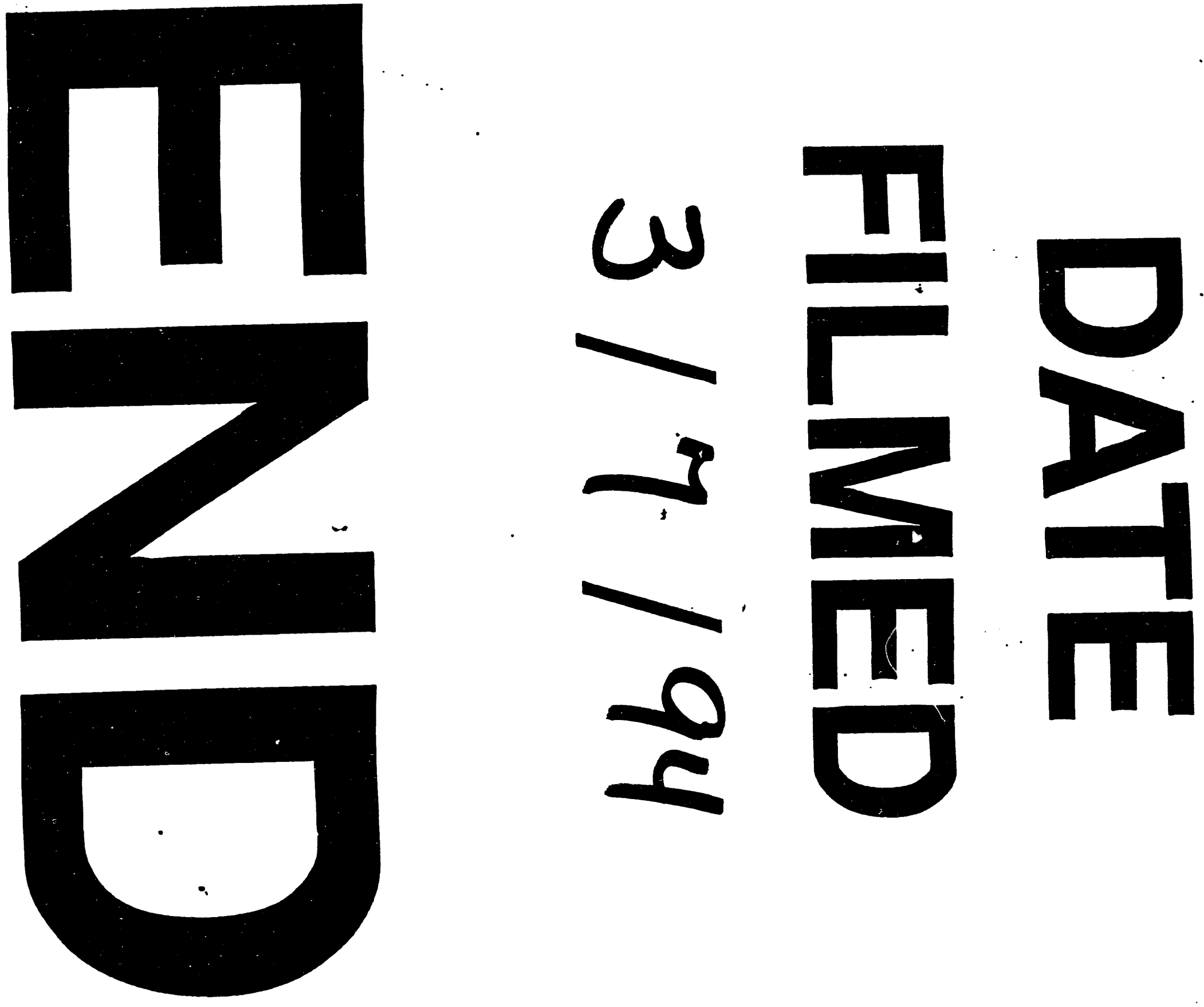